\title{
Erosion of a granular bed driven by laminar fluid flow
}

\author{
A LEXANDER E. L OBKOVSK Y , ASHISH V. ORPE , $^{2}$ \\ RYA N MOLLOY ARSHAD KUDROLLI \\ AND DANIEL H. ROTHMA N ${ }^{1}$ \\ ${ }^{1}$ Department of Earth, Atmospheric, and Planetary Sciences, Massachusetts Institute of \\ Technology, Cambridge, MA 02139 \\ ${ }^{2}$ Physics Department, Clark University, Worcester, MA 01610
}

(Received 24 October 2018)

\begin{abstract}
Motivated by examples of erosive incision of channels in sand, we investigate the motion of individual grains in a granular bed driven by a laminar fluid to give us new insights into the relationship between hydrodynamic stress and surface granular flow. A closed cell of rectangular cross-section is partially filled with glass beads and a constant fluid flux $Q$ flows through the cell. The refractive indices of the fluid and the glass beads are matched and the cell is illuminated with a laser sheet, allowing us to image individual beads. The bed erodes to a rest height $h_{r}$ which depends on $Q$. The Shields threshold criterion assumes that the non-dimensional ratio $\theta$ of the viscous stress on the bed to the hydrostatic pressure difference across a grain is sufficient to predict the granular flux. Furthermore, the Shields criterion states that the granular flux is non-zero only for $\theta>\theta_{c}$. We find that the Shields criterion describes the observed relationship $h_{r} \propto Q^{1 / 2}$ when the bed height is offset by approximately half a grain diameter. Introducing this offset in the estimation of $\theta$ yields a collapse of the measured Einstein number $q^{*}$ to a power-law function of $\theta-\theta_{c}$ with exponent $1.75 \pm 0.25$. The dynamics of the bed height relaxation are well described by the power law relationship between the granular flux and the bed stress.
\end{abstract}

\section{Introduction}

The response of a granular bed to forcing by a fluid which flows through and over the bed has been the subject of continuous inquiry for over a century (see, for example Graf (1971); Yalin (1977)). This phenomenon is at the centre of a wide range of practical and fundamental problems. Predicting the granular flux for a known fluid flow is important in understanding how beaches (Bailard 1981; Komar 1998), rivers (Murray \& Paola 1994) and deltas (Kenyon \& Turcotte 1985; Parker et al. 1998) evolve, mountains erode (Burbank et al. 1996) and landscapes form (Howard 1994). Sedimentary records cannot be deciphered without a working understanding of the combined fluid/granular (two-phase) flow (Blum \& Tornqvist 2000). The microscopic details of the bed's response to forcing by a fluid raise important fundamental questions about the nature of fluid flow near a rough (Grass 1971; Jimenez 2004) and/or permeable (Brinkman 1949; Beavers \& Joseph 1967) wall, the motion of a grain on a rough surface (Samson et al. 1999; Quartier et al. 2000), and the dynamics of granular avalanches (Douady et al. 2002; Bonamy et al. 2002).

Given some measure of the fluid flow intensity, one would like to predict the granular 
flux from the properties of the granular material such as size, shape, friction coefficient, bed packing, etc. An important aspect of the problem to consider first is the onset of granular flow. Just as the surface of a granular pile driven by gravity alone relaxes to the angle of repose (Jaeger et al. 1989), granular beds driven by fluid flow are thought to be static below a certain threshold fluid flux. Several empirical curves relating some measure of the fluid forcing at the onset of granular flow to the grain properties have been proposed (Shields 1936; Vanoni 1964; Yalin 1977). Numerous experimental studies aimed at computing these curves (summarised in Miller et al. (1977) or Buffington \& Montgomerv (1997), for example) are difficult to interpret since measured critical properties (such as the Shields parameter) vary by as much as a factor of three from study to study. The disparate and subjective definitions of the onset of granular flow used by the researchers are frequently used to explain the observed scatter (Shvidchenko \& Pender 2000; Paphitis 2001).

Even the existence of the threshold fluid forcing below which the granular flux is identically zero is a subject of a lively ongoing debate (Graf \& Pazis 1977; van Riin 1989). When the flow is turbulent, the local bed forcing is stochastic (Papanicolaou et al. 2001) and a strong case, supported by data, can be made for the presence of a granular flux (albeit vanishingly small) for any mean value of the fluid forcing (van Rijn 1989). The omnipresence of a granular flux poses a two-fold problem. First, an arbitrary threshold grain flux (Neill \& Yalin 1969), dislodging rate (Shvidchenko \& Pender 2000), or dislodging probability (Dancev et al. 2002) must be introduced to characterise the onset. Whereas the study of Graf \& Pazis (1977) suggests that the granular flux is exponentially small below some threshold driving, no systematic attempt, to our knowledge, has been made to quantify the transition in the functional dependence of the granular flux on driving. Second, the ever-present granular flux results in the evolution of the surface packing. This "ageing" or "armouring" of the granular bed leads to a decrease in the granular flux and an increase in the perceived threshold for the onset of persistent granular flow (Charru et al. 2004; Paphitis \& Collins 2005).

Even when the flow is laminar, the rough surface of the granular bed yields a fluctuating local fluid stress, albeit deterministically related to the time dependent realization of the surface packing. Statistical methods are therefore necessary to completely characterise the onset of granular flow. Because we expect the surface packing to have a transient component, so will the granular flux. However, we expect there to be a true threshold forcing below which there exist surface packings such that the associated surface stress is insufficient to dislodge any grains. Under steady pre-threshold forcing conditions, one such surface packing will eventually be realized and the granular flow will cease. We are interested in predicting the value of the threshold fluid forcing, the transient granular flux in pre-threshold conditions, and the steady state granular flux above the threshold. We would also like to establish whether a single characteristic of the fluid forcing (such as the surface stress) is sufficient to predict the steady state granular flux.

Here we report our initial investigation of these questions in a cell partially filled with transparent spherical glass beads driven by an index-matched fluid. The small size of the cell and the grains and the high viscosity of the fluid ensures that the particle Reynolds number is always less than ten. Adding small fluorescing tracers and illuminating the cell with a laser sheet allows us to directly image all grains in a vertical slice through the system. We can therefore simultaneously measure the fluid and granular fluxes, the dimensions of the fluid filled region, and the granular packing. This allows us to test theoretical predictions of the threshold condition and the functional dependence of the granular flux on fluid driving. Our study may be considered complementary to that of 


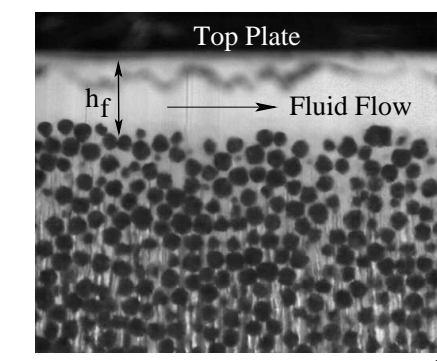

(b)

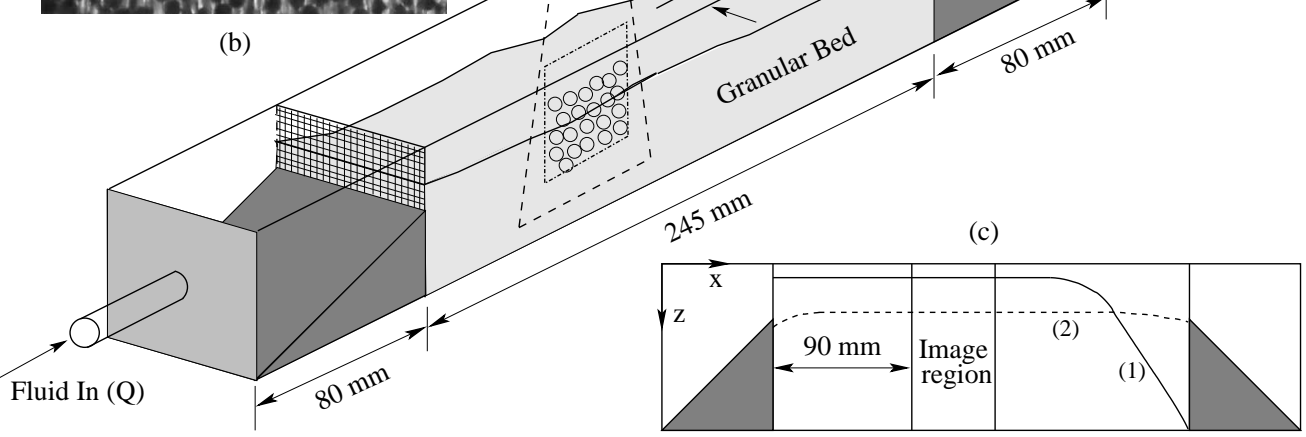

Figure 1. (a) Schematic diagram of the experimental apparatus. (b) Sample image of the refractive index matched beads in a plane away from the side walls. (c) Side view of the experimental apparatus. Solid line (1) is the initial profile of the granular bed after the preparation steps (i)-(iii) are carried out. Dashed line (2) is a sketch of typical profile of the granular bed after the cessation of erosion, the profile is perfectly flat except near the ends. The image region is located about $90 \mathrm{~mm}$ (130d) from the upstream end.

Goharzadeh et al. (2005), who examined the fluid flow near a granular bed using a similar setup.

\section{Experimental technique}

As shown in figure1, an enclosed cell with a rectangular cross-section is partially filled with glass beads with diameter $d=0.70 \pm 0.05 \mathrm{~mm}$, density $\rho_{g}=2.5 \mathrm{~g} / \mathrm{cm}^{3}$ and index of refraction of $n=1.54$. A peristaltic pump is used to generate a constant fluid flux $Q$ through the cell. The fluid is manufactured to have the same refractive index as the glass beads and a dye is added which fluoresces with a peak intensity at a wavelength of 565 $\mathrm{nm}$ when illuminated by light with a wavelength of $532 \mathrm{~nm}$ (Siavoshi et al. 2006). The hydrocarbon based fluid, manufactured by Cargille, Inc, has density $\rho_{f}=1.026 \mathrm{~g} / \mathrm{cm}^{3}$ and kinematic viscosity $\nu=0.243 \mathrm{~cm}^{2} / \mathrm{sec}$.

The granular bed is prepared using the following three steps. (i) we tilt the cell vertically with the fluid input end at the bottom, allow the grains to come to rest and (ii) slowly set it down horizontally. This procedure fills the cell top leaving an empty buffer space near the exit mesh (see figure 1 1 ). Step (iii) of bed preparation involves applying a small fluid flux to create a small (3-4 grain diameters) uniform gap. The three steps above are repeated to create the initial condition for all runs. We scanned the laser sheet across the initial bed surface and verified that it was flat in the observation window of width to within half a bead diameter. After the bed is prepared, the fluid flux is instantaneously raised to some value $Q$ and kept constant for the duration of each run. After traversing the pile's surface, eroded grains fall out of the way into the empty buffer space between the pile and the downstream mesh. Eventually, all granular motion ceases. In such a rest 
state the profile is perfectly flat except in regions of order $2 \mathrm{~cm}$ near the inlet and outlet meshes. We therefore suppose, and argue further below, that in the flat region, which comprises at least a $20 \mathrm{~cm}$ portion of the cell, the fluid flow profile is steady, laminar and independent of the downstream distance. We further checked that systematically varying the initialization fluid flux to create different initial bed heights did not change the final bed height. We observed that the final height reached was the same to within a grain diameter, provided the initial bed height was greater.

The range of fluid fluxes $Q$ for which the rest state is reached is a function of the cell filling fraction. If the cell filling fraction is high, the range of $Q$ is rather small. However, if the filling fraction is small, the quiescent bed does not have a flat region when the rest state is reached. We therefore set the uniform bed level at roughly $22 \mathrm{~mm}$.

A laser and cylindrical lens system, placed above the cell, illuminates a vertical slice through the cell away from the sidewalls. Because the beads do not contain the dye, they appear dark against a bright background fluid. A high speed digital camera with a resolution of $512 \times 480$ pixels records a sequence of images at a rate of 30-60 frames per second. A typical image of a vertical slice through the bed is shown in figure $1 \mathrm{~b}$. The image is truncated at a depth of about $15 \mathrm{~mm}$ (measured from the top plate) below which the particles never move. The apparent size of a bead depends on the distance of its centre from the illuminated plane. We are able to identify all beads whose centres are within approximately $0.3 d$ from the illuminated plane. The height of the granular bed in every image is measured using an edge detection algorithm. We take the height of the granular bed to be the inflection point in a depth profile of the pixel intensity averaged over the image width. This technique yields the mean height of the bed in the observation window. Furthermore, the mean height is averaged over 5 experimental runs to reduce statistical fluctuations, and remove any variability in preparing the initial pile.

We verified that the bed height decreases monotonically away from the side walls up to a distance of about $3.5 \mathrm{~mm}$ beyond which it remains constant to within the roughness scale of the granular bed. For all subsequent measurements we therefore positioned the laser sheet at a distance $7 \mathrm{~mm}$ from the side walls. The imaged region is about $16 \mathrm{~mm}$ wide, and is located $90 \mathrm{~mm}$ downstream from the inlet mesh (see figure1 $1 \mathrm{c}$ ). Comparing the height data for experimental runs repeated under same preparation conditions, we observed the standard deviations to be less that one particle diameter.

The inlet and outlet ramps ensure a smooth flow of the fluid onto the granular bed. After a process of trial and error, the height of the ramps $(22 \mathrm{~mm})$ was chosen to be slightly lower than the height of the granular bed when the grains fill the cell uniformly forming a flat surface. The leading edge of the bed tends to align with the top of the inlet ramp. If the bed is eroded below the upper edge of the inlet ramp, an undesirable turbulent region can form behind it. Neglecting end effects is theoretically justified because we find a relationship between the grain flux and the local viscous shear stress in a region away from both ramps. In addition, we find that the final rest height of the bed away from the ramps depends only on fluid flux $Q$ and not on the bed preparation or on the geometry of the ramps. Therefore, we maintain that the ramps only affect the granular bed in their vicinity and do not influence the bed height further than a certain distance $\ell_{b}$ downstream. To estimate $\ell_{b}$, we identify the length scale at which the width $\delta(x)$ of the Blasius boundary layer equals half the fluid depth $h_{f}$. The characteristic flow velocity is $U \sim Q / W h_{f}$, where $W$ is the width of the cell. Therefore

$$
\ell_{b} \sim \frac{h_{f}^{2} U}{\nu} \sim \frac{Q h_{f}}{4 W \nu} .
$$

For the largest values of the flux $Q$ and the fluid depth $h_{f}$ in our experiment, the length 


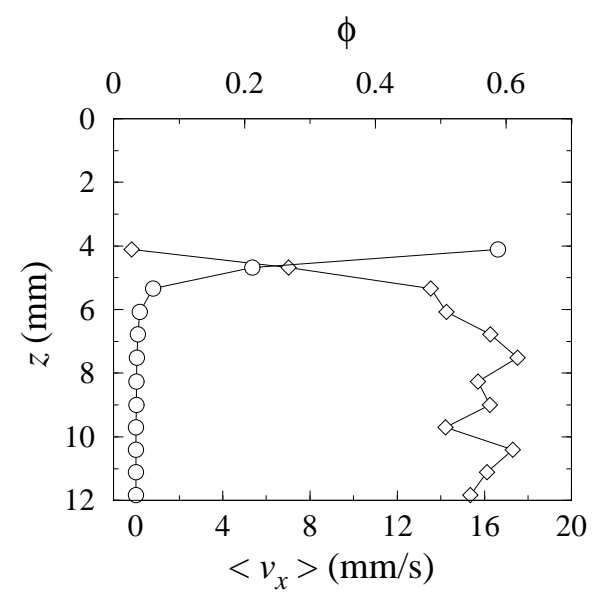

FIGURE 2. The horizontal granular velocity $\left\langle v_{x}\right\rangle$ (bottom axis, circles), computed in the regime when grains are rolling on the surface of a quiescent pile, averaged over the observation window and a one second time interval. The granular volume fraction $\phi$ is plotted with diamonds (top axis). The vertical axis denotes the depth $z$ measured from the cell's top plate; $z=0$ corresponds to the top plate; the bed's surface is located where $\phi$ vanishes. The total granular flux is proportional to the $z$ integral of the product of $\left\langle v_{x}\right\rangle \phi$.

$\ell_{b} \approx 100 d$. Our use of the laminar flow profile in the window of observation located $130 d$ downstream from the inlet ramp is therefore justified.

To compute the vertical profile of the particle velocity and packing fraction we measure the position of every particle in the image to within $0.1 d$ using a centroid technique (Siavoshi et al. 2006; Tsai et al. 2003). The velocity $v_{x}$ of individual particles is then determined by tracking the particles over two successive images. The image region is divided into horizontal bins of height $1 d$. The volume fraction $\phi$ is determined by dividing the number of particles in each bin by the volume of the bin $(1 d \times 0.6 d \times 22 d)$. The depth dependence of the bin-averaged and time averaged (over approximately one second) velocity $\left\langle v_{x}\right\rangle$ and volume fraction $\phi$ for one particular flow rate is shown in figure2, Above a certain depth no grains are detected in the bin which yields a null packing fraction. Given the depth profiles of the horizontal velocity and volume fraction, the grain flux $q_{g}$ (averaged over the observation window) is obtained by computing the integral $\int\left\langle v_{x}\right\rangle \phi d z$. Every reported flux value represents the average over a time interval of one second within which the variation in the flux values is small. All the experiments were repeated five times and the reported values are the averages over these five runs.

Before we launch into the detailed analysis of the data, let us qualitatively describe the phenomenon. Immediately after the fluid flow is switched on, the granular bed experiences a brief period (a few seconds) of rapid shear flow during which several grain layers are moving. The fluid gap increases rapidly during the shear flow regime. Subsequently, the granular flux is conveyed via "bed-load", i.e. individual grains rolling on the surface of an apparently quiescent bed. After several minutes the granular flux eventually vanishes. Because the erosion is gradual, the fluid gap increases gradually. Thus, the bead shear stress decreases gradually and the bed (away from the ramps) must approach a trivial flat state in which the fluid exerts a threshold shear stress on the bed. 

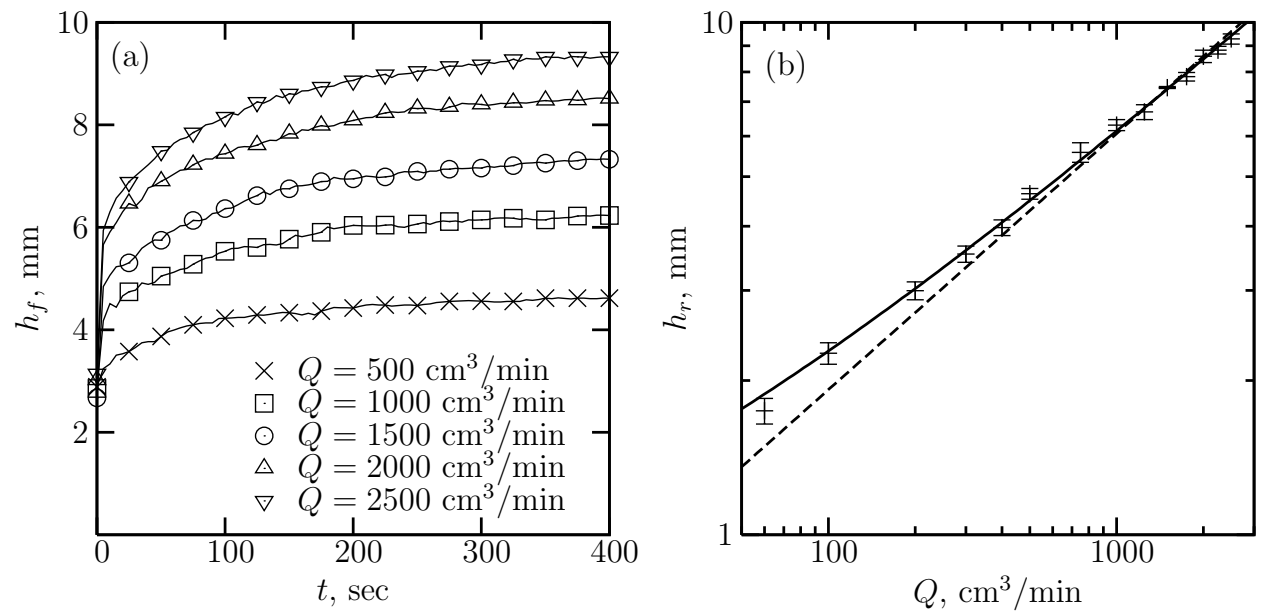

Figure 3. (a) The average depth $h_{f}$ of the fluid layer above the granular bed. Solid lines are a guide to the eye. (b) The rest depth $h_{r}$ of the fluid layer measured after cessation of granular flow as a function of fluid flux $Q$. Dashed line is a fit to equation (3.2). Solid line is the same fit with $h_{r}$ offset by roughly $0.4 \mathrm{~mm}$. For reference, the fluid depth above the entrance ramp is $11 \mathrm{~mm}$.

\section{Rest height of the granular bed}

Figure 3 a shows the average depth of the fluid gap as a function of time for a constant fluid flux $Q$ over the bed. As the grains are eroded from the bed's surface and deposited into the buffer space, the fluid gap grows, and the mean fluid flow speed and therefore the shear stress on the bed decreases. The erosion eventually ceases and the fluid gap approaches a constant rest depth $h_{r}$ which decreases with the applied fluid flux $Q$.

The gradually increasing fluid gap and thus gradually decreasing bed stress leads to the cessation of erosion precisely at the threshold driving. The variation of the rest depth $h_{r}$ with the applied fluid flux $Q$ allows us to examine the validity of the Shields threshold criterion. We estimate the bed shear stress $\sigma$ assuming two-dimensional laminar flow between infinite smooth parallel plates separated by $h_{f}$. This approximation is good when the cell width $W \approx 26 \mathrm{~mm}$ is much greater than the fluid gap depth $h_{f}$. Using the solution for laminar flow in a pipe of rectangular cross-section (see for example Cornish (1928)) we can compute the correction to the estimated bed stress. Neglecting the sidewalls results in an underestimate of the stress by $8 \%$ for the smallest fluid gap and by $29 \%$ for the largest fluid gap in our experiment. However, neglecting the sidewalls has the advantage of a simple analytic expression for the bed stress which greatly simplifies the analysis. The laminar flow assumption is good since the cell Reynolds number for $Q=1000 \mathrm{~cm}^{3} / \min$ is $\operatorname{Re}=v_{\text {mean }} h_{f} / \nu=Q / W \nu \approx 0.3$, where $v_{\text {mean }}$ is the horizontal fluid velocity averaged over the fluid gap. The Shields parameter $\theta$, defined as the stress $\sigma$ scaled by the hydrostatic pressure difference $\left(\rho_{g}-\rho_{f}\right) g d$ across the grain, is

$$
\theta=\frac{6 Q \nu}{W \gamma g d h_{f}^{2}}
$$

where $\gamma \equiv \rho_{g} / \rho_{f}-1 \approx 1.437$ is the density contrast. The Shields parameter measures the relative importance of the destabilising hydrodynamic forces and the stabilising gravity.

Assuming that the rest depth $h_{r}$ of the fluid gap corresponds to the Shields criterion 


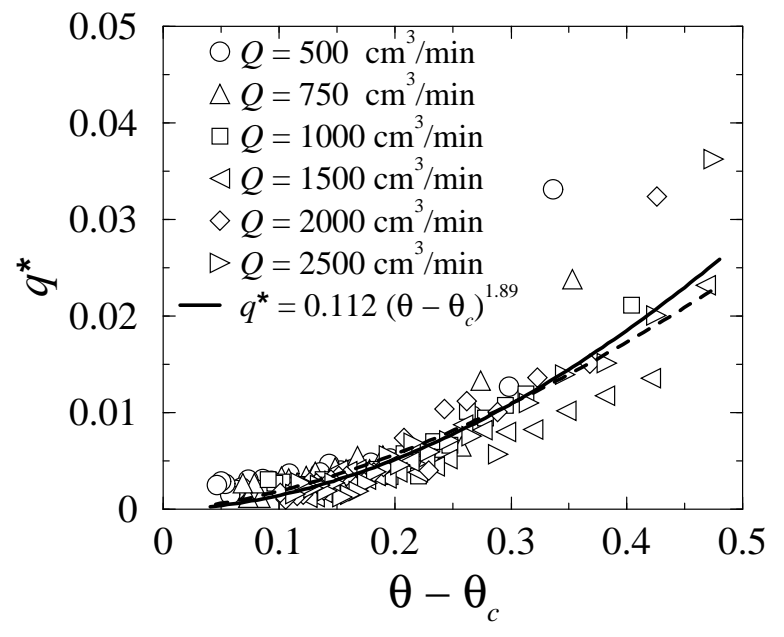

FiguRE 4 . Einstein number $q^{*}$ vs. the excess $\theta-\theta_{c}$ of the dimensionless shear stress. Solid line is a power-law fit to the region $\theta-\theta_{c}<0.3$. Dashed line is a power law fit with $\lambda=1.6$ fixed.

$\theta=\theta_{c}$, we obtain

$$
h_{r}=d\left(\frac{Q}{Q_{r}}\right)^{1 / 2}, \quad \text { with } \quad Q_{r}=\theta_{c} \frac{W \gamma g d^{3}}{6 \nu} .
$$

The fit to the measured rest depth (shown as a dashed line in figure $3 \mathrm{~b}$ ) is significantly improved if a positive constant of approximately half a diameter $d$ is introduced to the right-hand side of (3.2) (an even better fit is obtained if $h_{r} \sim Q^{0.45}$ ). The fit yields $Q_{r}=15.4 \pm 0.3 \mathrm{~cm}^{3} /$ min which translates to the critical Shields parameter of $\theta_{c}=0.30 \pm 0.01$. This value is consistent with previously reported values, for example, in Miller et al. (1977) or Buffington \& Montgomery (1997). For comparison, the Yalin parameter, $\Xi \equiv\left(\gamma g d^{3}\right)^{1 / 2} / \nu$, a less widely used dimensionless group which measures the relative importance of viscous and gravitational forces without reference to the flow intensity, is $\Xi \approx 0.03$ in our experiment.

The physical origin of the offset is not entirely clear to us at this time. The correction to the bed stress estimate due to the sidewalls cannot be the source of the offset since in the limit of small gap $h_{f}$ the sidewall effect vanishes. A possible source of the offset is the fluid boundary condition at a permeable wall which relates the derivative of the fluid velocity to its magnitude at some penetration length scale $\ell$. Yet another possibility is that the offset could be due to the way in which $h_{f}$ is measured. We use the mean surface height to represent the mean boundary stress. In reality, however, fluid flow past a rough permeable medium creates a distribution of bed stresses and the estimate of its mean using a non-slip boundary condition at a flat wall is a gross approximation. The fact that the error in the estimate of the mean stress translates to a height offset that is only half a bead diameter is a pleasant surprise.

\section{Flow rule}

Tracking all grains in a vertical slice as mentioned in section 2 allows us to directly measure the grain flux $q_{g}$ and to attempt to relate it to the estimated mean bed stress. In general, other factors besides bed stress, such as the flow history, could influence the 
grain flux. Although an unambiguous relationship between grain flux and bed stress is usually tacitly assumed, here we test it directly.

Following convention (Einstein 1950) we use the Einstein number $q^{*}=q_{g} /\left(\gamma g d^{3}\right)^{1 / 2}$, i.e. the non-dimensionalised grain flux. Figure 4 is a plot of $q^{*}$ vs. the estimated excess bed stress $\theta-\theta_{c}$ computed using equation (3.1) with the value of $\theta_{c}=0.3$ measured in Section 3 Different symbols correspond to different fluid fluxes $Q$. Because the same bed stress occurs at different fluid gap depths for different $Q$ and therefore entirely different times in the history of the erosion process, data collapse suggests that only the instantaneous bed stress (and not its history, for example) is required to predict the instantaneous grain flux. Therefore, upon changes (in time or space) of the bed stress the grain flux quickly relaxes to its corresponding value. We hypothesise from dimensional considerations that the relaxation time scale is $d^{2} / \nu \approx 0.02 \mathrm{sec}$, which is small indeed.

The data in Figure 4 do not collapse for high stresses, i.e. early times, because small fluid gaps are associated with changes in the mechanisms of granular transport. At early times the granular flux occurs primarily as bulk shear of the granular pile, whereas later in the history of bed evolution the grain flux is conveyed through isolated grains rolling over the otherwise immobile granular bed. The latter form of transport is known as the "bed-load" regime. A power law fit to the data collapse yields

$$
q^{*} \approx A\left(\theta-\theta_{c}\right)^{\lambda}, \quad \text { with } \quad \lambda=1.89 \pm 0.25, \quad A=0.11 \pm 0.03 .
$$

Our measured exponent $\lambda$ is slightly higher than the widely accepted value of 1.5 (Mever-Peter \& Müller 1948), and also above the value of 1.6 found by Wong \& Parker (2006) in their reanalysis of Mever-Peter \& Müller (1948). The pre-factor $A$, however, is more than an order of magnitude smaller than that quoted by Wong \& Parker (2006) for turbulent flow. The large discrepancy suggests that although the granular flux in the laminar fluid flow regime is described by the same functional dependence on the Shields parameter as in the turbulent regime, the pre-factor is not. The turbulent fluid appears to be significantly more efficient at entrainment and transport of surface grains. This is perhaps not surprising since for the same mean bed stress, the turbulent bursts provide a more effective dislodging process than the smoothly varying bed stress in the laminar flow regime.

\section{Approach to rest height}

The experimentally verified relationship between the bed stress and the granular flux allows us to compute the entire history of the fluid gap depth $h_{f}(x, t)(x$ is the coordinate in the flow direction) as it approaches the rest depth $h_{r}$. The mean thickness of the flowing grain layer is negligible compared to $h_{f}$ in the "bed-load" regime. Therefore, we write the conservation of sediment as

$$
\frac{\partial h_{f}}{\partial t}=\frac{\partial q_{g}}{\partial x} .
$$

To study the approach of the profile to the rest height $h_{r}$, we introduce $\xi=h_{f} / h_{r}$, scale all lengths by $d$ and times by $\sqrt{d / \gamma g}$ and use the flow rule (4.1) which can be expressed as $q^{*}=A \theta_{c}^{\lambda}\left(1 / \xi^{2}-1\right)^{\lambda}$. Eq. (5.1) can therefore be written as

$$
\frac{\partial \xi}{\partial t}+c(\xi) \frac{\partial \xi}{\partial x}=0, \quad c(\xi)=2 \lambda A \theta_{c}^{\lambda} \xi^{-1-2 \lambda}\left(1-\xi^{2}\right)^{\lambda-1} \frac{d}{h_{r}} .
$$

Given an initial condition $\xi\left(x, t_{0}\right)=\xi_{0}(x)$, the solution to equation (5.2) (obtained by the method of characteristics) determines $\xi(x, t)$ implicitly through $\xi(x, t)=\xi_{0}(x-$ $c(\xi(x, t)) t)$.

There are two issues which make the full solution of (5.2) difficult to use. First, a 
possibility exists that the granular flux includes a term proportional to the gradient of the bed height resulting in a diffusive term in (5.2). Our measurements of the bed height are not sufficiently resolved to verify or reject such a diffusive term. Second, the domain of applicability of equation (5.2) is limited in time and space. In the beginning of the experiment, granular transport is mainly conveyed via bulk shear and thus does not obey (4.1). Also, near the inlet ramp the bed height may change rapidly and thus the approximate expression for the shear stress (3.1) is invalid there. Fixing the initial and boundary conditions for equation (5.2) is therefore not feasible. Thus the full solution is not useful.

However, the long time asymptotic solution to (5.2) with a small diffusive term may be insensitive to the initial and boundary conditions. Therefore, we seek a separable solution to (5.2) in the limit $\xi \rightarrow 1$ (i.e. nearly flat bed)

$$
\xi(x, t)=1-\left(\frac{x-x_{0}}{B\left(t-t_{0}\right)}\right)^{1 /(\lambda-1)},
$$

where $x_{0}$ and $t_{0}$ are integration constants and $B=\lambda A\left(2 \theta_{c}\right)^{\lambda} d / h_{r}$.

Because the height of the bed changes by less than a diameter along the cell, an experimental test of the spatial variation in Eq. (5.3) is impractical in our setup. We therefore focus on the approach of the fluid depth averaged over the observation window to the rest depth $h_{r}$ (cf. figure 3a). Equation (5.3) implies that once the fluid gap depth $h_{f}$ is scaled by rest depth $h_{r}$ and time is scaled by $h_{r} / \sqrt{\gamma g d}$, the data should collapse onto a master curve which approaches unity as the power law $\left(t-t_{0}\right)^{1 /(1-\lambda)}$. Figure 5 shows the data collapse. The power law fit to the resulting data cloud yields the offset time $t_{0} \approx-2.2 h_{r} / \sqrt{\gamma g d}$ and the exponent $\lambda=1.6 \pm 0.1$. The predicted data collapse suggests that the assumed functional relationship between the instantaneous bed stress and granular flux is indeed correct. It is difficult to interpret the value of $t_{0}$, since the asymptotic solution (5.3) only applies at long times. The fitted value for $\lambda$ falls just outside the $1 \sigma$ interval of the value quoted in (4.1), but is consistent with the value found by Wong \& Parker (2006).

\section{Discussion}

In summary, we have revisited the well-studied problem of a sand bed driven by an overlying viscous fluid. We focused on precise measurements in the laminar flow regime where little data is available. Our flow geometry, in which the depth of the fluid gap gradually increases as the grains are eroded, results in the cessation of granular flow and thus allows for an unambiguous definition of the threshold condition. Therefore, by varying the fluid flux $Q$ through the cell, we have verified the hypothesis that the boundary viscous shear stress is sufficient to predict cessation of granular flow over the surface of a horizontal granular surface. The directly measured value of the critical Shields parameter $\theta_{c}=0.30 \pm 0.01$ and the Yalin parameter $\Xi=0.03$ is consistent with the extrapolation of the previously reported values of $\theta_{c}$ to this value of $\Xi$. The advantage of approaching the threshold from above is that there is no ambiguity or subjectivity in its definition. On the other hand, a relatively rapid approach to the quiescent state in our setup precludes a thorough study of the "armouring" phenomenon. Finally, we remark that the threshold for the cessation of granular flow may differ from that for the onset of granular flow. This effect is difficult to quantify in general since the onset of granular flow is marked by transient flow and steady driving is impossible in our cell since any erosion leads to a decrease in driving. 


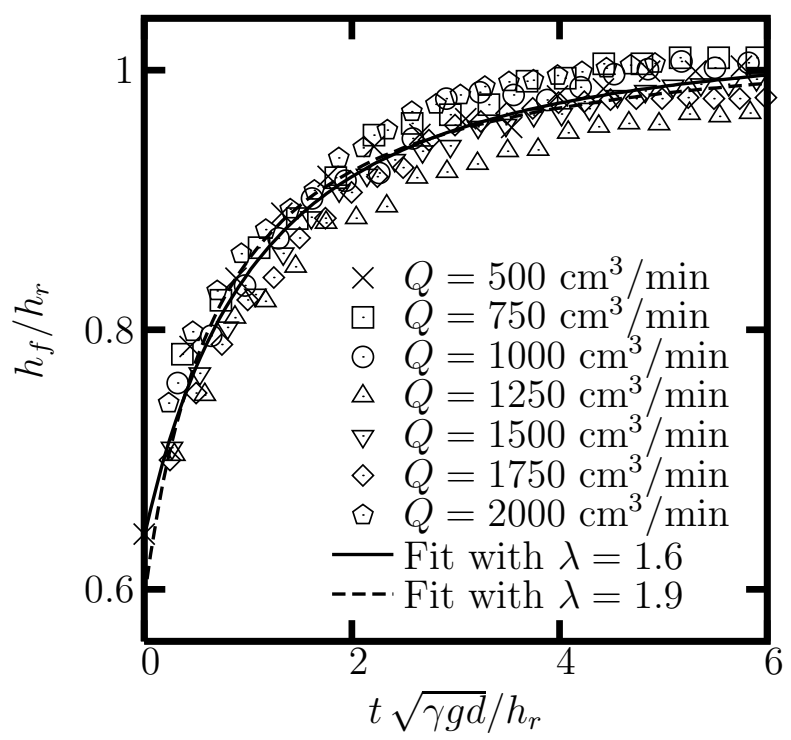

FiguRE 5. Fluid gap depth scaled by the rest depth $h_{r}$ vs. time scaled by $h_{r} / \sqrt{\gamma g d}$. Solid line is a fit to a function that approaches unity as $\left(t-t_{0}\right)^{1 /(1-\lambda)}$ with $\lambda=1.6$ and $t_{0}=-2.2 h_{r} / \sqrt{\gamma g d}$. For comparison, we include a fit to a power law with $\lambda=1.9$ (dashed line).

We directly verified that the boundary shear stress determines not only the onset of granular flow, but also the granular flux over a roughly horizontal bed in non-steady driving conditions. This is an important result since it implies that the granular flux quickly adjusts to changes in the driving. We have established the power law relationship between the grain flux and the excess boundary shear stress via two independent measurements. We measure the granular flux directly by particle tracking and, alternatively, we analyse the approach of the bed height to the quiescent state. The average of the two independently measured exponents is $\lambda=1.75 \pm 0.25$. The pre-factor in the power law relationship between the Einstein number and the excess Shields stress is more than an order of magnitude smaller than that in the widely used "bed-load" granular flux rule (Wong \& Parker 2006), indicating perhaps a different mechanism for the granular transport in the laminar fluid flow regime.

We have measured the parameters in the granular flux rule (4.1) not only for a particular range of Reynolds numbers, but also for a particular type of grains - smooth spheroids. How the parameters in (4.1) depend on the grain properties such as shape, friction constant and roughness is an interesting and open question.

Lastly, we note that since the threshold condition in our experiment is reached gradually, our problem is similar to the relaxation of a dry granular pile expressed in terms of the angle of repose. Moreover, the concept of a Shields threshold, typically defined via the onset of granular flow due to gradually increased fluid driving, may be analogous to the notion that dry granular piles can be characterised by a maximum angle of stability.

The work was funded by the Department of Energy grants DE-FG0202ER15367 (Clark), DE-FG0299ER15004 (MIT), and the National Science Foundation grant number CTS0334587 (Clark). 
BAILARD, J. A. 1981 An energetics total load sediment transport model for a plane sloping beach. J. Geophys. Res.-Oceans and Atmospheres 86 (NC11), 938-54.

Beavers, G. S. \& Joseph, D. S. 1967 Boundary conditions near a permeable wall. J. Fluid Mech. 30 (1), 197-207.

Blum, M. D. \& Tornqvist, T. E. 2000 Fluvial responses to climate and sea-level change: a review and look forward. Sedimentology 47, 2-48.

Bonamy, D., Daviaud, F., Laurent, L., Bonetti, M. \& Bouchaud, J. P. 2002 Multiscale clustering in granular surface flows. Phys. Rev. Lett. 89 (3), 034301.

Brinkman, H. C. 1949 A calculation of the viscous force exerted by a flowing fluid on a dense swarm of particles. Appl. Sci. Res. A 1 (1), 27-34.

Buffington, J. M. \& Montgomery, D. R. 1997 A systematic analysis of eight decades of incipient motion studies, with special reference to gravel-bedded rivers. Water Resour. Res. 33 (8), 1993-2029.

Burbank, D. W., Leland, J., Fielding, E., Anderson, R. S., Brozovic, N., Reid, M. R. \& DuncAn, C. 1996 Bedrock incision, rock uplift and threshold hillslopes in the northwestern Himalayas. Nature 379, 505-10.

Charru, F., Mouilleron, H. \& Eiff, O. 2004 Erosion and deposition of particles on a bed sheared by a viscous flow. J. Fluid Mech. 519, 55-80.

Cornish, R. J. 1928 Flow in a pipe of rectangular cross-section. Proc. Roy. Soc. London, Ser. A 120 (786), 691-700.

Dancey, C. L., Diplas, P., Papanicolaou, A. \& Bala, M. 2002 Probability of individual grain movement and threshold condition. J. Hydraul. Eng. 128 (12), 1069-75.

Douady, S., Andreotti, B., Daerr, A. \& Clade, P. 2002 From a grain to avalanches: on the physics of granular surface flows. Compt. Rend. Phys. 3 (2), 177-86.

Einstein, H. A. 1950 The bed-load function for sediment transportation in open channel flows. Technical Bulletin 1026. United States Department of Agriculture, Washington.

Goharzadeh, A., Khalili, A. \& Jorgensen, B. B. 2005 Transition layer thickness at a fluid-porous interface. Phys. Fluids 17, 057102.

Graf, W. H. 1971 Hydraulics of sediment transport. McGraw-Hill, New York.

Graf, W. H. \& PAZIS, G. C. 1977 Deposition and erosion in an alluvial channel. J. Hydraul. Res. 15, 151-65.

Grass, A. J. 1971 Structural features of turbulent flow over smooth and rough boundaries. $J$. Fluid Mech. 50, 233-55.

HowARD, A. D. 1994 A detachment-limited model of drainage-basin evolution. Water Resour. Res. 30 (7), 2261-85.

JAeger, H. M., Liu, C. \& NAgel, S. R. 1989 Relaxation at the angle of repose. $P R L 62$ (1), 40-43.

Jimenez, J. 2004 Turbulent flows over rough walls. Ann. Rev. Fluid Mech. 36, 173-196.

Kenyon, P. M. \& Turcotte, D. L. 1985 Morphology of a delta prograding by bulk sediment transport. Geol. Soc. Am. Bull. 96, 1457-65.

Komar, P. D. 1998 Beach processes and sedimentation. Prentice-Hall, Upper Saddle River, N.J.

Meyer-Peter, E. \& Müller, R. 1948 Formulas for bed-load transport. In Proceedings of the 2nd Meeting of the International Association for Hydraulic Structures Research, pp. 39-64. Int. Assoc. Hydraul. Res., Delft, Netherlands.

Miller, M. C., McCave, I. N. \& Komar, P. D. 1977 Threshold of sediment motion under unidirectional currents. Sedimentology 24, 507-27.

Murray, A. B. \& PAOlA, C. 1994 A cellular-model of braided rivers. Nature 371, 54-7.

Neill, C. R. \& YAlin, M. S. 1969 Quantitative definition of beginning of bed movement. J. Hydraul. Div. Am. Soc. Civ. Eng. 95, 585-8.

Papanicolaou, A. N., Diplas, P., Dancey, C. L. \& Balakrishnan, M. 2001 Surface roughness effects in near-bed turbulence: Implications to sediment entrainment. J. Eng. Mech.ASCE 127 (3), 211-8.

PAPhitis, D. 2001 Sediment movement under unidirectional flows: an assessment of empirical threshold curves. Coastal Engineering 43, 227-45.

Paphitis, D. \& Collins, M. B. 2005 Sand grain threshold, in relation to bed 'stress history': an experimental study. Sedimentology 52, 827-38. 
Parker, G., Paola, C., Whipple, K. X. \& Mohrig, D. 1998 Alluvial fans formed by channelized fluvial and sheet flow. i: Theory. J. Hydraul. Eng.-ASCE 124 (10), 985-95.

Quartier, L., Andreotti, B., Doundy, S. \& Daerr, A. 2000 Dynamics of a grain on a sandpile model. Phys. Rev. E 62 (6), 8299-8307.

VAN RiJn, L. C. 1989 Handbook of sediment transport by currents and waves. Report H 461. Delft Hydraulics, Delft, Netherlands.

Samson, L., Ippolito, I., Bideau, D. \& Bartouni, G. G. 1999 Motion of grains down a bumpy surface. Chaos 9 (3), 639-48.

ShIELDS, A. 1936 Anwendung der Ähnlichkeitsmechanik und der Turbulenzforschung auf die Geschiebebewegung. Tech. Rep. Heft 26. Mitteilung der Preussischen Versuchsanstalt für Wasserbau und Schiffbau, Berlin, Germany. (In German).

Shvidchenko, A. B. \& Pender, G. 2000 Flume study of the effect of relative depth on the incipient motion of uniform sediments. Water Resour. Res. 36 (2), 619-28.

Siavoshi, S., Orpe, A. V. \& Kudrolli, A. 2006 Friction of a slider on a granular layer: Nonmonotonic thickness dependence and effect of boundary. Phys. Rev. E $\mathbf{7 3}$ (1), 010301.

Tsai, J.-C., Voth, G. A. \& Gollub, J. P. 2003 Internal granular dynamics, shear-induced crystallization, and compaction steps. Phys. Rev. Lett. 91 (6), 64301.

VANONI, V. A. 1964 Measurements of critical shear stress for entraining fine sediments in a boundary layer. Tech. Rep. KH-R-7. W. M. Keck Lab. of Hydraulics and Water Resourses, Cal. Inst. Tech.

Wong, M. \& PARKer, G. 2006 Reanalysis and correction of bed-load relation of Meyer-peter and Müller using their own database. J. Hydraul. Eng. 132 (11), 1159-68.

Yalin, M. S. 1977 Mechanics of sediment transport, 2nd edn. Pergamon Press, Oxford, New York. 